\title{
Spatial Uniformity of Interface Trap Distribution in MOSFET's
}

\author{
NELSON S. SAKS, Senior member, IEEe, AND MARIO G. ANCONA, MEMber, ieEe
}

\begin{abstract}
The uniformity of the spatial distribution of (fast) interface traps $N_{i t}$ in small MOS devices has been determined using charge pumping on MOSFET's with varying lengths and widths. The number of traps is found to be linearly proportional to both length and width as expected for a macroscopically uniform distribution. No evidence is found for an anomalous $N_{i t}$ distribution at the edges of the source/drain regions; however, the data suggest that there is a higher density of traps along the edges of LOCOS field oxides.
\end{abstract}

\section{INTRODUCTION}

$F^{\prime}$ AST-INTERFACE traps $N_{i t}$ are electrically active defects which exist at $\mathrm{Si}-\mathrm{SiO}_{2}$ interfaces of metal-oxide-semiconductor (MOS) devices where they cause degradation in device performance. In an MOS field-effect transistor (MOSFET), large $N_{i t}$ densities can cause increased leakage currents, higher noise, and decreased MOSFET transconductance due to reduced free-carrier surface mobility.

In the past, much of the research on interface traps in MOS devices has been obtained using capacitance-voltage ( $C-V$ ) measurements on relatively large (typically $\geq$ $100 \mu \mathrm{m})$ devices. Although small-scale spatial variations (surface potential fluctuations) have been studied extensively in large capacitors [1], $N_{i t}$ is generally assumed to be macroscopically uniform. This assumption becomes questionable when devices are small $(0.1-10 \mu \mathrm{m})$ since the influence of device edges can become significant. Edge regions may have different $N_{i t}$ densities because, for example, various device fabrication steps may introduce laterally nonuniform damage. High densities of crystalline defects may be created near the source/drain regions during ion implantation, especially for relatively heavy ions such as arsenic [2]. It is well known that high densities of substrate defects exist at field oxide edges when using LOCOS (local oxidation of silicon [3]) field isolation [4][6], which could also cause higher interface trap densities in these regions. Dry (reactive-ion) etching of polycrystalline silicon ("polysilicon") gates in MOS structures may cause surface damage and/or contamination along the gate edges (similar to damage created by low-energy ion implantation) which may not be completely annealed during subsequent high-temperature processing [7]. Chin and

Manuscript received June 5, 1989; revised November 13, 1989. The review of this paper was arranged by Associate Editor R. R. Troutman.

The authors are with the Naval Research Laboratory, Code 6813, Washington, DC 20375

IEEE Log Number 8933688
Ma [8] and Zekeriya and Ma [9] have shown that the $N_{i t}$ density in irradiated aluminum-gate capacitors increases with width in the range $2-800 \mu \mathrm{m}$, but no dependence was observed before irradiation. This effect was ascribed to stress associated with the metal gate. Finally, the $N_{i t}$ density in actual MOS devices is normally reduced to manageable levels by a final high-temperature $\left(400-450^{\circ} \mathrm{C}\right)$ fabrication step which is typically performed in a partial hydrogen ambient for polysilicon-gate MOS devices. Since hydrogen diffusion rates are different in the polysilicon gate and the gate oxide, the edge regions will be exposed to different hydrogen concentrations which may lead to different $N_{i t}$ concentrations.

It is difficult to measure $N_{i t}$ uniformity in small MOS devices because most techniques either do not have sufficient sensitivity and/or simply measure average values. Charge pumping, a sensitive electrical technique for measuring $N_{i t}$ in MOSFET's, has been used with some success by several authors to measure nonuniform $N_{i t}$ distributions in MOSFET's damaged by hot carrier injection [10]-[14]. Recently, Plossu and coworkers [13] used charge pumping to measure the spatial distribution of $N_{i t}$ near the source-drain regions of n-channel MOSFET's without purposely introduced damage. They concluded that a significantly higher (more than ten times background) $N_{i t}$ density exists at these edges. If correct, this effect would be expected to strongly impact device performance.

In this work, we have assessed $N_{i t}$ uniformity by measuring the number of traps as a function of the MOSFET area. We use charge pumping in MOSFET's with different lengths and widths rather than, as in [10]-[14], a single MOSFET where the effective charge-pumping area was modulated by varying the source/drain reverse bias. In addition, we have performed measurements on devices obtained from several different sources, including commercial vendors, in an attempt to draw some general conclusions regarding "typical"' $N_{i t}$ edge distributions.

In summary, we find that interface traps appear uniformly distributed across the center of the MOSFET over both the length and width in all technologies measured. Furthermore, we do not find any observable nonuniformity near the edges of the source/drain regions. This result is in disagreement with results of Plossu et al. [13] discussed above. However, we do find that a higher $N_{i t}$ density appears to exist along all LOCOS field oxide edges, 
but not along field oxide edges which are defined by etchback of a thick, uniformly grown oxide. We suggest that either the high stress and strain of the LOCOS structure, or the off- $\langle 100\rangle$ orientation of the edge transition region (often called the "bird's beak"), results in a higher interface trap density in LOCOS transition regions.

\section{Sample Preparation and Experimental TECHNIQUES}

The test devices used were fabricated at NRL and two commercial vendors and are summarized in Table I. Two of the technologies are representative of relatively old, large-geometry technologies (NRL PMOS1/5 and MOSIS/M64), while the other two are reasonably current $1.2-\mu \mathrm{m}$ gate length technologies (NRL NMOS2/4 and CMOS1 from a commercial IC manufacturer). The CMOS1 devices were fabricated with a complete 1.2- $\mu \mathrm{m}$ CMOS process, but only the n-channel devices were tested. CMOS1/6 has abrupt arsenic junctions, while CMOS $1 / 2$ is identical except for a shallow lightly doped drain $\left(L_{D D}\right)$ structure to control hot carrier effects. The NRL devices are intended to be test devices and consequently are not representative of a complete process; for example, these devices lack the usual overcoat glass passivation layer. All the devices were fabricated using mask designs from NRL. These designs include blocks of MOSFET's with different lengths and widths suitable for charge pumping and transconductance measurements which are located close together on the die to minimize wafer-scale variations in characteristics. Typically such variations in $N_{i t}$ were less than $\pm 15 \%$ in these wafers. In the following experiments, we assume that different size MOSFET's have identical physical structure except for the size of the active regions, which appears consistent with actual results.

The number of interface traps $N_{i t}$ in a given MOSFET was obtained using the constant-amplitude ac signal technique described by Groeseneken et al. [15]. In this technique, an ac signal of constant amplitude (typically 2-3 $\mathrm{V}$ ) is applied to the MOSFET gate as shown in Fig. 1. The MOSFET substrate is held at ground potential. The charge pumping current $I_{c p}$ is measured at the source and drain (tied together) or, alternatively, at the substrate, as a function of the dc offset voltage ( $\equiv V_{\text {low }}$ ) of the ac signal. The maximum $I_{c p}$ is given by [15]

$$
I_{c p}(\max )=q f N_{i t}
$$

where $f$ is the frequency of the ac signal, $q$ is the electron charge, and $N_{i t}$ is the number of interface traps. The amplitude of the ac signal must be large enough to swing the surface potential in the MOSFET active region from accumulation to inversion and therefore varies with oxide thickness; a typical value is $3 \mathrm{~V}$ for $t_{\mathrm{ox}} \leq 30 \mathrm{~nm}$. In this work we have used an ac signal of $100 \mathrm{kHz}$ with $1-\mu$ s rise and fall times. For this ac signal, the $N_{i t}$ values are an average over approximately the central $0.6 \mathrm{eV}$ of the silicon bandgap. $N_{i i}$ distributions as a function of energy have also been measured using a different but related
TABLE I

Summary OF Process LotS

\begin{tabular}{|c|c|c|c|c|c|c|c|}
\hline MANUFACTURER & L LOT ID & TECHNOLOGY & $\begin{array}{l}t_{\text {ox }} \\
(\mathrm{nm})\end{array}$ & $\begin{array}{c}\text { FIELD } \\
\text { ISOLATION }\end{array}$ & IYPE & $\begin{array}{l}\text { OURCE/DF } \\
\text { JUNCTION } \\
\text { DOPANT } \\
\end{array}$ & $\begin{array}{l}\text { RAIN } \\
\text { IS } \\
\frac{\text { DEPTH }}{(\mu \mathrm{m})}\end{array}$ \\
\hline NRL & NMOS2/4A & $1.25 \mu \mathrm{m}$ NMOS & 22 & LOCOS & $\mathbf{N}^{+}$ & As & 0.2 \\
\hline NRL & PMOS1/5 & $4 \mu \mathrm{m}$ PMOS & 26 & ETCHED & p* & B & $>0.5$ \\
\hline COMMERCIAL & $\begin{array}{l}\text { CMOS } 1 / 2 \\
\text { CMOS1/6 }\end{array}$ & $\{1.25 \mu \mathrm{m}$ cMOs $\}$ & $\begin{array}{l}25 \\
25\end{array}$ & $\begin{array}{l}\text { Locos } \\
\text { LOcOS }\end{array}$ & $\begin{array}{l}\mathbf{N}^{+} \\
\mathbf{N}^{+}\end{array}$ & $\begin{array}{l}P\left(L_{D D}\right) \\
A s\end{array}$ & $\begin{array}{l}<0.2 \\
\sim 0.2\end{array}$ \\
\hline FOUNDRY & MOSIS/M64 & $3-4 \mu \mathrm{m}$ NMOS & 61 & Locos & $\mathbf{N}^{+}$ & $\mathbf{P}$ & $>0.5$ \\
\hline
\end{tabular}

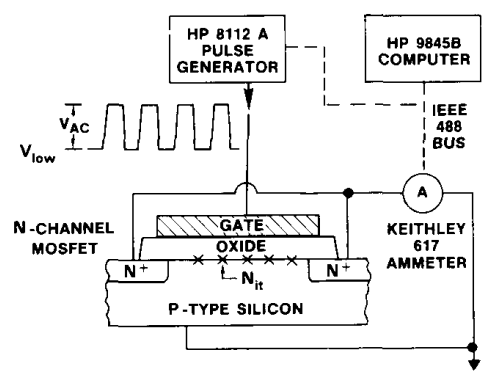

Fig. 1. Experimental charge-pumping setup for measuring $N_{i t}$ in MOSFET's.

charge-pumping technique involving changing the rise and fall times of the ac signal [15]. In general, we find that $N_{i t}$ varies by a factor of about $2-3$ over the central $0.8-\mathrm{eV}$ energy range which can be probed, but the details of the distributions are different for each technology. The charge-pumping techniques used to make these measurements are described in greater detail elsewhere [14], [16]. All charge-pumping measurements reported here have been obtained on MOSFET's with gate lengths less than $15 \mu \mathrm{m}$. Longer lengths were found to lead to erroneous pumping currents due to geometric effects [15]. All measurements here were made at room temperature $(\approx$ $296 \pm 3 \mathrm{~K}$ )

In the experiments below, charge-pumping data will be compared with transconductance measurements obtained on the same MOSFET's. The obvious advantage of using identical devices is that, when different devices are used for the two measurements, it may be questionable whether the devices are really the same. The effective MOSFET length $\left(L_{\text {eff }}\right)$ and width ( $\left.W_{\text {eff }}\right)$ are obtained from the MOSFET drain current $I_{d}$ as a function of the gate voltage $V_{g}$ in the triode regime [17]

$$
\begin{aligned}
I_{d} & =\frac{k_{0}\left(V_{g}-V_{t}-V_{d} / 2\right) V_{d}}{\left(1+\theta\left(V_{g}-V_{t}\right)\right)} \\
k_{0} & =\mu_{0} C_{\mathrm{ox}} W_{\mathrm{eff}} / L_{\mathrm{eff}}
\end{aligned}
$$

where $V_{g}$ is the gate voltage, $V_{t}$ is the threshold voltage, $V_{d}$ is the applied drain voltage $(50-100 \mathrm{mV}), \mu_{0}$ is the (low-field) mobility, $C_{\text {ox }}$ is the gate oxide capacitance, $\theta$ is a parameter which accounts for the effects of source/ drain series resistance [17] and also reduced surface mobility due to the gate oxide electric field, and $W_{\mathrm{eff}} / L_{\mathrm{eff}}$ is the ratio of effective width to length. A least squares fit of 
TABLE II

Summary of Effective Length and Width Data

\begin{tabular}{|c|c|c|c|c|c|c|c|c|c|c|}
\hline $\begin{array}{c}\text { PROCESS } \\
\text { I.D. }\end{array}$ & $\begin{array}{c}\text { MOSFET } \\
\text { WIDTH } \\
(\mu \mathrm{m})\end{array}$ & $\begin{array}{l}\text { \#CHIPS } \\
\text { TESTED }\end{array}$ & $\begin{array}{l}\Delta L_{1 d} \\
(\mu m)\end{array}$ & $\begin{array}{l}\Delta L_{c p} \\
(\mu \mathrm{m})^{\circ}\end{array}$ & $\begin{array}{c}\text { MOSFET } \\
\text { LENGTH } \\
(\mu \mathrm{m})\end{array}$ & $\begin{array}{l}\text { \#CHIPS } \\
\text { TESTED }\end{array}$ & $\begin{array}{c}\Delta W_{1 d} \\
(\mu m)\end{array}$ & $\begin{array}{l}\Delta W_{\phi p} \\
(\mu m)\end{array}$ & $\begin{array}{c}\bar{N}_{4 k} \\
\left(\times 10^{10}\right. \\
\left.\text { TRAPS/(cm }{ }^{2}\right) \\
\end{array}$ & $\begin{array}{c}\text { EDGE } \\
N_{\mathrm{H}} \\
\text { (TRAPS } / \mu \mathrm{m})\end{array}$ \\
\hline NMOS2/4A & 150 & 3 & 1.21 & {$\left[\begin{array}{ll}1.38 & (\mathrm{OV}) \\
1.48 & (2 \mathrm{~V})\end{array}\right]$} & 15 & 4 & 3.16 & 1.92 & 5.5 & 310 \\
\hline NMOS2/4A & 15 & 4 & 0.97 & 1.30 & & & & & 5.2 & \\
\hline PMOS1/5 & 150 & 4 & 1.94 & 2.14 & 15 & 4 & 0.78 & 1.03 & 0.77 & $\sim 0$ \\
\hline $\begin{array}{l}\text { CMOS1/2 } \\
\text { CMOS1/6 }\end{array}$ & $\begin{array}{l}25 \\
25\end{array}$ & $\begin{array}{l}1 \\
4\end{array}$ & $\begin{array}{l}0.63 \\
0.85\end{array}$ & $\begin{array}{l}1.20 \\
1.24\end{array}$ & $\begin{array}{l}10 \\
10\end{array}$ & $\begin{array}{l}3 \\
3\end{array}$ & $\begin{array}{l}+0.37 \\
+0.20\end{array}$ & $\begin{array}{l}-0.60 \\
-0.63\end{array}$ & $\begin{array}{l}2.0 \\
1.6\end{array}$ & $\begin{array}{r}120 \\
64\end{array}$ \\
\hline MOSIS/M64 & 15 & 2 & 0.50 & 1.00 & 15 & 4 & 0.20 & 0.01 & 0.65 & 15 \\
\hline
\end{tabular}

at source/drain bias of ov EXcept as noted in parentheses

the $I_{d}-V_{g}$ data to (2) is performed to obtain the fitting parameters $k_{0}, V_{t}$, and $\theta$. In most of the data below, each data point represents an average of several devices from nearby chips to reduce the possible effects of chip-to-chip variations (the number of devices tested is listed in Table II). Distributions of experimental transconductance data were reasonably tight (typically $\pm 2 \%$ except in the very small devices), indicating little nonuniformity. Somewhat larger variation was always found in the $N_{i t}$ values (typically $\pm 5 \%$ or so, depending on process lot and wafer). Since the $N_{i t}$ variation was not due to nonuniformity in the average area (this would have shown up in the transconductance data), the $N_{i t}$ variation is probably due to true chip-to-chip variations in interface trap densities.

\section{Experimental Results And Discussion}

\section{A. $N_{i t}$ versus MOSFET Length}

In Fig. 2, $N_{i t}$ and transconductance $1 / k_{0}$ values are plotted against the drawn (i.e., the dimension drawn on the photoresist mask) MOSFET gate length for a series of n-channel MOSFET's from lot CMOS1/6 with identical width. The same devices are used for both $I_{c p}$ and transconductance measurements. Each data point represents an average of four devices from adjacent chips. As shown in Fig. 2(a), $N_{i t}$ increases linearly with MOSFET length, which is consistent with a uniform $N_{i t}$ distribution from source to drain. Even the data for the smallest MOSFETs in Fig. 2 fall on the same straight line, indicating that any source/drain edge nonuniformity is confined to distances smaller than half the smallest effective length tested, or about $0.4 \mu \mathrm{m}$. Similar results showing $I_{c p}$ increasing linearly with gate length have recently been reported by Hofmann and Krautschneider [18]. For the transconductance measurements on the same MOSFET's (Fig. 2(b)), 1/ $k_{0}$ is also found to be proportional to the drawn gate length, in agreement with (2).

An interesting feature of the data in Fig. 2 is that the $x$ axis intercepts for $N_{i t}$ and transconductance are not the same. The $x$ axis intercept $(\equiv \Delta L)$ should be the drawn length at which the actual (or effective) active MOSFET length is zero. The effective gate length $L_{\mathrm{eff}}$ for any given

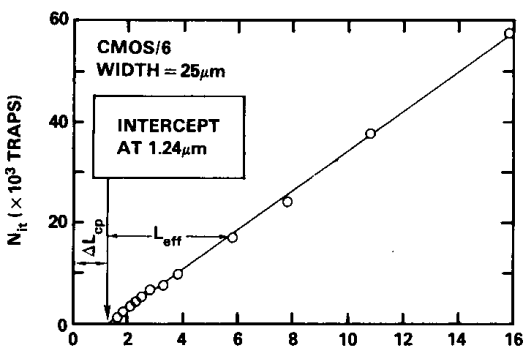

(a)

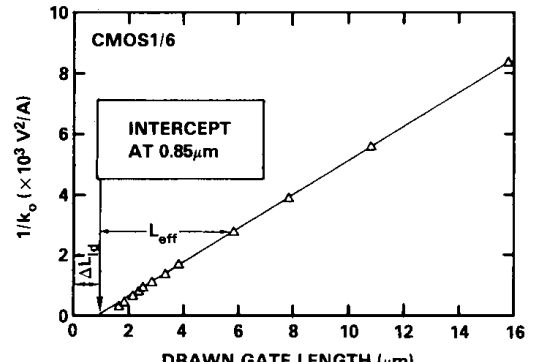

(b)

Fig. 2. (a) $N_{i}$ measured by charge pumping as a function of drawn gate length for a series of MOSFET's with different gate length (but constant width) from lot CMOS1/6. Solid lines are best least square fits of the data to straight lines. The linear dependence of $N_{i f}$ on gate length is consistent with a uniform $N_{\text {is }}$ spatial distribution. The $x$-axis intercept is the difference between the drawn and effective gate lengths. (b) $1 / k_{0}$ (an electrical parameter determined by fitting to the MOSFET $I_{d}-V_{g}$ characteristic, defined by (2)) versus drawn MOSFET gate length. The $x$-axis intercept is the difference between the drawn and effective gate lengths for conventional electrical characterization. Each data point for $N_{i t}$ and $1 / k_{0}$ is an average from four MOSFET's, and the same devices are used for both measurements.

MOSFET is equal to its drawn gate length minus $\Delta L$, as depicted in Fig. 2(b). $\Delta L$ values in Fig. 2 are $+1.24 \pm$ 0.1 and $+0.85 \pm 0.05 \mu \mathrm{m}$ for $\Delta L_{c p}$ and $\Delta L_{I d}$, respectively (the error in $\Delta L_{I d}$ is smaller because of the smatler scatter in the transconductance measurements). Both of the effective lengths are smaller than the drawn length. This is primarily due to shrinkage of the polysilicon gate during various fabrication steps (mainly resist definition and etching), and lateral diffusion of the source/drain junctions under the gate. 


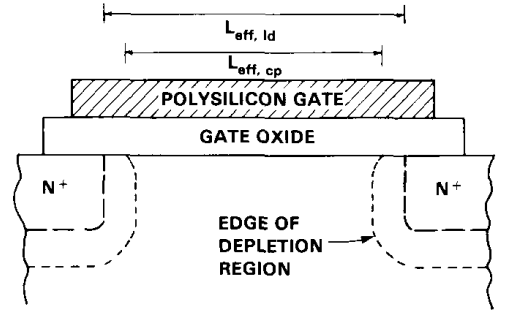

Fig. 3. Schematic cross section of an n-type MOSFET showing the existence of depletion regions at the edges of the $n^{+}$source and drain. Interface traps within these depletion regions at the oxide surface cannot be charge-pumped because holes are excluded from these regions. The effective MOSFET length for charge pumping $\left(L_{\text {eff }}\right)$, therefore, is smaller than the effective length $\left(L_{\mathrm{eff} .} . d\right)$ obtained from conventional $I_{d d^{-}}$ $V_{g}$ measurements

In Fig. 2, the effective length for charge pumping is smaller than that for transconductance in a given MOSFET. Indeed, $\Delta L_{l d}$ is smaller than $\Delta L_{c p}$ for all technologies measured, as shown in Table II. This likely arises because of the different nature of the two measurements. As shown in the MOSFET cross section in Fig. 3, $L_{\mathrm{eff}, I d}$ is (very nearly) equal to the distance between the source and drain metallurgical junctions. $L_{\mathrm{eff} . . p}$ is somewhat different. In order for a given interface trap to contribute to the charge-pumping signal, the local interface must alternate between strong accumulation and strong inversion. This trap then alternately fills with electrons and holes whose recombination gives rise to the charge pumping signal. In an n-channel MOSFET in accumulation, holes are excluded from the depletion regions associated with the $\mathrm{n}^{+}$source/drain regions, and thus traps in these regions cannot contribute to $I_{c p}$. Therefore, $L_{\mathrm{eff} . c p}$ is smaller than $L_{\mathrm{eff}, I d}$ by the sum of the widths of the surface depletion regions at the source and drain.

To see if this explanation quantitatively fits our results, similar measurements of $N_{i t}$ and $1 / k_{0}$ versus drawn gate length are shown for NRL n-channel MOSFET's in Fig. 4 (ignore for the moment the curve labeled $V_{r}=2 \mathrm{~V}$ ). The NRL devices are used here because the detailed structural information necessary to calculate the lateral depletion region widths was not available for the non-NRL devices. Both $N_{i t}$ and $1 / k_{0}$ in Fig. 4 are linearly proportional to gate length, in agreement with previous results on the CMOS $1 / 6$ devices. The $x$-axis intercepts $(=\Delta L$ values $)$ are summarized in Table II. For these devices, $\Delta L_{c p}$ is $1.38 \pm 0.1 \mu \mathrm{m}$ compared to $\Delta L_{I d}$ at $1.21 \pm 0.05 \mu \mathrm{m}$. Thus the effective length of these devices is $0.17 \pm 0.15$ $\mu \mathrm{m}$ smaller for charge pumping compared to transconductance. To compare this value with theory, two-dimensional (2-D) computer simulations of the surface hole carrier density have been obtained using MINIMOS [19] as a function of position near the MOSFET drain with gate voltage as a parameter. Similar calculations have recently been used by Heremans et al. [20] to calculate the effect of the proximity of the source and drain on the full chargepumping characteristic. Dopant profiles in the $\mathrm{n}^{+}$(as shown at the bottom of Fig. 5) and channel regions which

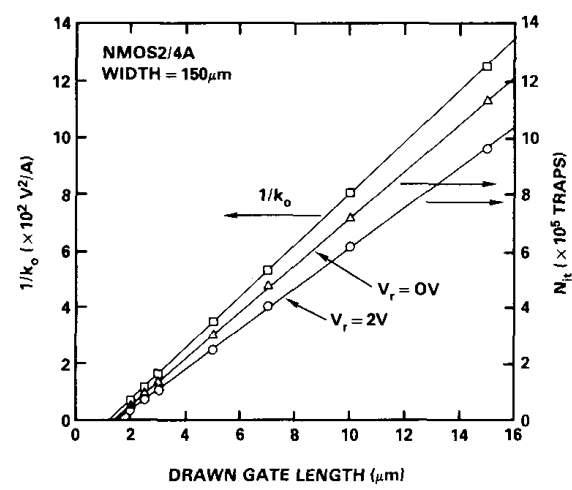

Fig. 4. $N_{i t}$ and $1 / k_{0}$ as a function of drawn gate length for MOSFET's from lot NMOS $2 / 4 \mathrm{~A}$. The solid lines are least square fits to the data. Measurements were obtained with 0 - and $2-\mathrm{V}$ reverse bias applied to the source and drain. Application of this reverse bias increases the size of the lateral depletion region which decreases $L_{\mathrm{t} f \mathrm{f}, \mathrm{p}}$ by a small amount (see Table II)

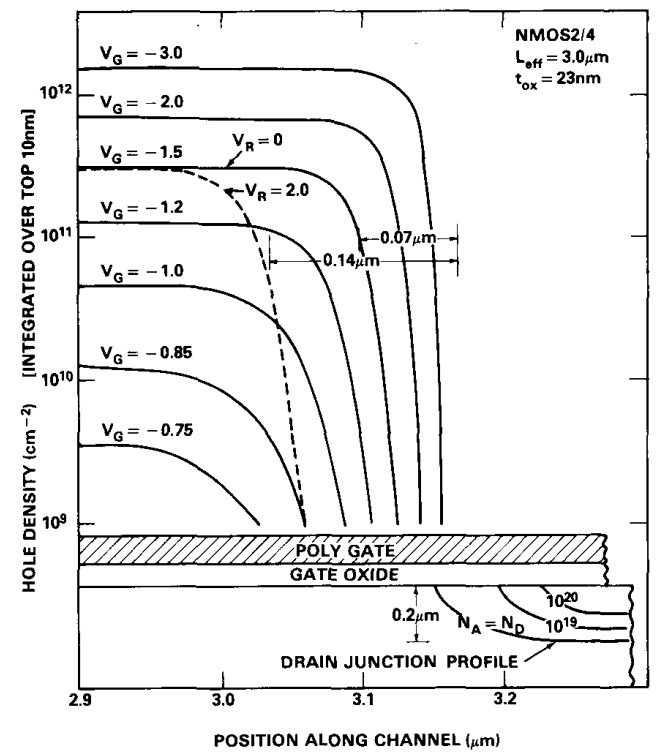

Fig. 5. Plot of surface hole density as a function of position near the drain (or source) for several gate biases. A MOSFET cross section on the same lateral scale, including the drain junction doping profile, is shown at the bottom. The data are calculated using the 2-D numerical simulation program MINIMOS. Calculations are shown for zero bias on the drain (solid lines) and 2-V reverse bias (dashed line). The lateral surface depletion region (as defined for charge pumping-see text) increases from 0.07 to $0.14 \mu \mathrm{m}$ by applying $2-\mathrm{V}$ bias.

are required for these calculations were obtained from a combination of process simulations and SIMS data. These profiles have been used by us extensively in other modeling calculations on similar samples and are believed to be reasonably accurate. As shown in Fig. 5, the density of holes decreases rapidly close to the $n^{+}$diffusion. We define the edge of the depletion region which is effective for charge pumping as the position where the surface density of free holes (in units of holes per square centimeter) 
is equal to the interface trap density. ${ }^{1}$ The average $N_{i t}$ value for the NMOS $2 / 4 \mathrm{~A}$ devices is equal to $5.5 \times 10^{10}$ traps $/ \mathrm{cm}^{2}$ over $0.6 \mathrm{eV}$, or $1.0 \times 10^{11}$ traps $/ \mathrm{cm}^{2}$ integrated over the entire 1.1-eV silicon bandgap, assuming that $N_{i t}$ is constant versus energy. The position of the drain edge was determined from 2-D simulations as the position at which the surface electron concentration does not significantly change with gate bias. This position is slightly inside the drain metallurgical junction at $N_{A}=N_{D}$. From these calculations, a surface-depletion region of $0.07 \mu \mathrm{m}$ is determined for the actual charge-pumping biases used in this experiment, which results in an effective gate length for charge pumping which is $0.14 \mu \mathrm{m}$ smaller than that for transconductance. This agrees reasonably well with the experimental difference of $0.17 \pm 0.15 \mu \mathrm{m}$, especially considering the relatively large margin of error.

On the NMOS2/4A wafers, a second set of MOSFET's with smaller gate width ( 15 compared to $150 \mu \mathrm{m}$ ) were also available. For completeness, measurements were also made on these devices. Results are shown in Table II. In this case the $\Delta L$ values are somewhat smaller than the $150-\mu \mathrm{m}$ width MOSFET's in Fig. 4, and the difference between $L_{\mathrm{eff} . I d}$ and $L_{\mathrm{eff} . c p}$ is significantly larger $(0.33$ $\pm 0.15 \mu \mathrm{m})$. We believe that the larger difference is caused primarily by corner effects which may be significant in these relatively narrow MOSFET's, especially considering the large bird's beak regions at the field oxide edge ( $\Delta W$ is greater than $3 \mu \mathrm{m}$ in these MOSFET's). Some of the difference may also be caused by variation across the wafer (the 15- and 150- $\mu \mathrm{m}$ devices were from different regions of the wafer) as shown by the large variation in $L_{\mathrm{eff}, I d}$.

Experimental charge pumping and transconductance effective length values are summarized in Table II for the four different technologies tested in this work. In every case, the shrinkage in $L_{\mathrm{eff}}$ is larger for charge pumping than for transconductance, though the difference is relatively small and difficult to measure $(0.17-0.57 \mu \mathrm{m} \pm$ $0.15 \mu \mathrm{m})$. This result supports the concept shown in Fig. 3 that the charge pumping $L_{\text {eff }}$ is smaller because $N_{i t}$ pumping does not occur in the depletion regions near the source/drain edges.

Further evidence that this explanation is correct is obtained by increasing the widths of the lateral depletion regions by applying a reverse bias $V_{r}$ to the source and drain diffusions during the charge-pumping measurement. A large reverse bias should increase the width of the depletion region and therefore decrease the effective pumping length. On the other hand, measurement of the transconductance effective length should not be, and experimentally is not, significantly affected by application of a source/drain bias. Computer simulations of surface

\footnotetext{
'It is possible to make other choices of the charge pumping "edge." For example, in [20, eq. (3)], the edge is defined as the position where trap capture time equals the time allowed during the ac signal. However, because of the very steep slope of the hole density in the edge region (Fig. 5), the calculated width of the edge depletion region will not be a stron function of the hole density chosen to define the edge.
}

hole densities for $V_{r}=2 \mathrm{~V}$ are shown in Fig. 5 for just the one value of gate bias $\left(V_{g}=-1.5 \mathrm{~V}\right)$ used during the pumping measurement. The simulation shows that the width of one depletion region increases to $0.14 \mu \mathrm{m}$. Experimental data in Fig. 4 shows that $\Delta L_{c p}$ with $V_{r}=2 \mathrm{~V}$ increases to $1.48 \pm 0.10 \mu \mathrm{m}$ from $1.38 \pm 0.10 \mu \mathrm{m}$ at $V_{r}$ $=0 \mathrm{~V}$. Thus the experimental difference in effective lengths at $V_{r}=2 \mathrm{~V}$ is $0.27(1.48-1.21) \pm 0.15 \mu \mathrm{m}$, in good agreement with the calculated value of $0.28(2 \times$ 0.14 ) $\mu \mathrm{m}$. The slope of the $N_{i t}$ data in Fig. 4 also changes with $V_{r}$ and is smaller at $2 \mathrm{~V}$ compared to $0 \mathrm{~V}$. This is not an indication that the interface trap density has changed but rather that the region of the bandgap over which the $N_{i t}$ are integrated is reduced from about $0.6 \mathrm{eV}$ to about $0.52 \mathrm{eV}$ due to the changed biasing conditions [15].

As discussed above, Plossu and coworkers [13] recently claimed to observe a large increase in $N_{i t}$ near the source/drain edges. They obtained this $N_{i t}$ spatial distribution by varying the source/drain reverse bias $V_{r}$ during charge-pumping measurements. For a $10-\mu \mathrm{m}$ gate length MOSFET, they found that $D_{i t}$ has a value larger than $1 \times$ $10^{11}$ traps $/ \mathrm{cm}^{2} \cdot \mathrm{eV}$ within about $1 \mu \mathrm{m}$ of the source/ drain edges as compared with less than $1.0 \times 10^{10}$ traps $/ \mathrm{cm}^{2} \cdot \mathrm{eV}$ in the center of the channel. From these values, the total number of edge defects is approximately $2 \times 10^{11}$ traps $/ \mathrm{cm} \cdot \mathrm{eV}$, which is a factor of two larger than the $1 \times 10^{11}$ traps $/ \mathrm{cm} \cdot \mathrm{eV}$ for a uniform distribution over the whole gate length. In contrast, in this work we find that the $N_{i t} x$-axis intercept (Fig. 4) is within approximately $0.2 \mu \mathrm{m}$ of the calculated intercept. Thus the maximum number of excess source/drain edge defects in a 10- $\mu \mathrm{m}$ length MOSFET must be less than about $0.2 / 10$, or only $2 \%$ of the total number of defects. In support of this argument, Ancona et al. [14] performed chargepumping profiling measurements on virgin and stressed MOSFET's similar to those in [13]. Although they also observed a larger variation in $I_{c p}$ with $V_{r}$ than would have been obtained for a spatially uniforms $N_{i t}$ in the virgin devices, they concluded that this effect is an artifact which is eliminated when the reverse bias is applied only during inversion (described in [14] as the "constant field" charge pumping technique). ${ }^{2}$

\section{B. $N_{i t}$ versus MOSFET Width}

It is also possible to determine the spatial uniformity of the $N_{i t}$ distribution across the MOSFET width by measuring $N_{i t}$ in an array of MOSFET's with different widths but constant length. Data for this experiment for both $k_{0}$ and $N_{i t}$ are shown in Fig. 6 for FET's from process lot NMOS2/4A. $k_{0}$ rather than $1 / k_{0}$ (as in Fig. 2) is plotted against drawn gate width since $I_{d}$ is proportional to effective width (see (2)). Once again, $N_{i}$ is linearly propor-

${ }^{2}$ In [14], the effect of reverse bias on the filling of the traps during accumulation was ascribed to the effect of the surface electric field on the trap occupancy. We have since realized that the trap filling would also be affected simply by the changing channel potential, and at present we cannot distinguish between the two. In either case, the effect is eliminated by holding the reverse bias constant during accumulation as was demonstrated by the "constant-field charge pumping" results in [14]. 


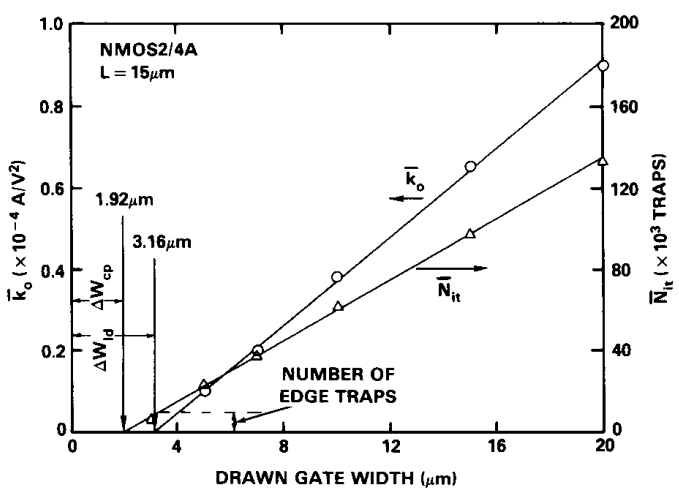

Fig. 6. $k_{0}$ and $N_{i}$ versus drawn gate width for a series of MOSFET's with different widths but the same length $(15 \mu \mathrm{m})$. Solid lines are the least squares fits to the data. Each data point is an average of four devices, and the same devices are tested in both measurements. The straight line behavior shows that $N_{i}$ scales linearly with MOSFET width. However, the $N_{i}$ intercept $(1.92 \mu \mathrm{m})$ is much smaller than the $k_{0}$ intercept. This is explained by assuming that the entire $N_{i t}$ curve is raised by a small (constant) number of traps in the edge regions which is found by assuming that the effective lengths for $N_{i t}$ and $k_{0}$ are identical.

tional to MOSFET area, implying that the $N_{i t}$ are uniformly distributed in the MOSFET active region.

An unusual aspect of the data in Fig. 6 is that the $N_{i t}$ intercept is smaller than the $k_{0}$ intercept by a very large amount, about $1.2 \mu \mathrm{m}$. How is this to be explained? A cross section through the width of an $n$-channel MOSFET (along the center of the polysilicon gate) is shown in Fig. 7 (a). Due to the $\mathrm{p}^{+}$doping under the field oxide (which helps prevent surface inversion in this region), a lateral depletion region exists between the electron inversion layer (when the MOSFET is turned on) and the $\mathrm{p}^{+}$substrate/field doping. The effective width $W_{\text {eff }}$ is defined by the size of the electron inversion layer as shown in Fig. 7(a). The transconductance $W_{\text {eff }}$ should be nearly identical to the charge pumping $W_{\text {eff }}$ since both are defined by the extent of this electron inversion layer (we estimate that the effective widths are the same within $\pm 0.1 \mu \mathrm{m}$ ). Thus an explanation similar to that used to explain differences in $L_{\text {eff }}$ will not be adequate for $W_{\text {eff }}$.

One possible explanation of the large apparent differences in effective widths observed in Fig. 6 is that there is a constant additive contribution to $N_{i t}$ from the edge regions, which would increase every $N_{i t}$ data point in Fig. 6 and result in an apparent decrease in effective width for charge pumping. Assuming this interpretation, $8.6 \times 10^{3}$ excess edge interface traps (measured by charge pumping) spread over $28 \mu \mathrm{m}\left(=2 * L_{\text {eff }}\right)$ of LOCOS edge in Fig. 6 , or 310 defects $/ \mu \mathrm{m}$ of edge, would be required to explain the discrepancy. It is likely that this significantly understates the actual numbers of interface traps in this region, since most of the surface in the transition region where the oxide is much thicker will not be charge pumped. Unfortunately, we do not know enough about the details of the structure and doping profiles in the LOCOS edge region of these MOSFET's to make a detailed calculation of the carrier densities, and of the area probed by charge pumping, in this region.

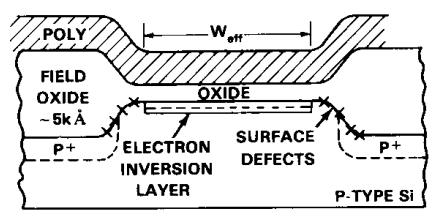

(a)

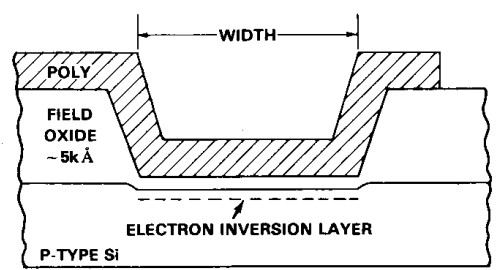

(b)

Fig. 7. (a) Schematic cross section across the width of an n-type MOSFET with conventional LOCOS field isolation. A lateral surface depletion region exists at the LOCOS edge between the $\mathrm{p}^{+}$field doping and the electron inversion layer. The effective MOSFET widths for both transconductance and charge pumping are defined by the lateral extent of the inversion layer; the two effective widths are therefore the same. (b) Cross section of MOSFET with etched field oxide. In this structure, the silicon step height and associated stress/strain are much smaller compared to LOCOS.

There are two possible reasons for an unusually high $N_{i t}$ density in the edge regions. First, it is well known that many bulk defects are created in LOCOS edge regions due to stresses induced by growth of the field oxide during fabrication [4]-[6]. It therefore seems reasonable that increased surface trap density may also exist in these regions as shown in Fig. 7(a). (The diagrams in Fig. 7 are conceptual, since the details of the structure and $N_{i t}$ distributions are not known.) Secondly, the orientation of the silicon substrate within the transition region from gate to field oxide is off of the $\langle 100\rangle$ orientation. Since the $N_{i t}$ density is generally smallest for $\langle 100\rangle$ orientation [21], the $N_{i t}$ density in the edge region is undoubtedly larger just due to the orientation difference. For a rough idea of the magnitude of this effect, we estimate that the $N_{i t}$ density in this off-orientation edge is five times larger, and that about $0.1 \mu \mathrm{m}$ (out of an estimated $0.5-\mu \mathrm{m}$ transition region) of the edge region is charge pumped. For NMOS2/ $4 \mathrm{~A}$, this results in a calculated edge trap density of 250 traps $/ \mu \mathrm{m}$, which is close to the measured value ( 310 traps $/ \mu \mathrm{m})$. At present, we cannot distinguish between these two causes, and in fact both may contribute to higher edge $N_{i t}$ in the actual devices.

Similar measurements of $W_{\text {eff }}$ for all the surveyed technologies are summarized in Table II. For devices from the commercial CMOS process, the charge-pumping effective width is much larger than that determined from transconductance, in qualitative agreement with the results on the NRL NMOS samples, again suggesting a high density of LOCOS-related edge defects. The foundry devices (MOSIS/M64), which are believed to employ a LOCOS field, have the same behavior but the difference in $W_{\text {eff }}$ is much smaller. This experimental result suggests that the edge trap density can be controlled by optimizing the processing. It is also interesting that the number of excess edge interface traps in LOCOS-isolated MOSFET's 
scales, at least approximately, with the average $N_{i t}$ in the center of the MOSFET gates, for the limited set of process lots tested here (see Table II).

If the existence of edge traps is related to the LOCOS processing as discussed above, then MOSFET's fabricated using a different field oxide process (e.g., without LOCOS) may not have different effective widths for transconductance and $N_{i t}$. To test this prediction, the field isolation in process lot PMOS1/5 was fabricated by uniform growth of a thick field oxide. The thick oxide was then removed from the active device areas by wet chemical etching. A cross section of the structure is shown in Fig. 7(b). There is still a small off-orientation step in the silicon substrate at the edge due to silicon consumption during growth of the gate oxide, but this step is much smaller (only about $150 \AA$ of $\mathrm{Si}$ is consumed). This technique for producing the field oxide is essentially without stress and consequently should introduce many fewer defects. As shown in Table II, the transconductance and $N_{i t}$ effective widths are the same within experimental error (about $\pm 0.2 \mu \mathrm{m})$. This result tends to confirm the idea that the smaller $\Delta W_{c p}$ obtained for the LOCOS devices as shown in Fig. 6 is indeed due to a higher density of edge interface traps.

\section{Summary}

The lateral distribution of interface traps $N_{i t}$ has been determined using charge pumping on MOSFET's with different lengths and widths. The area of the MOSFET which is effective for charge pumping is found to be slightly smaller than the area found by standard $I_{d}-V_{g}$ characterization. This is caused by lateral depletion regions at the source/drain edges during the accumulation cycle of the charge pumping measurement.

$N_{i t}$ is found to be distributed uniformly in the central area of the MOSFET between source and drain and between field oxide edges for all technologies surveyed. No significant increase in interface trap density was observed near the source/drain edges, in contrast to a recent report by Plossu et al. [13]. However, a much higher trap density was observed near the field oxide edges in two technologies which use the LOCOS field oxide process. Higher $N_{i t}$ densities in the edge regions could be caused by either stress/strain in the LOCOS structure which results in more bulk and surface defects, or by a higher density of surface traps due to the non- $\langle 100\rangle$ orientation of the LOCOS transition edge. The observed correlation between excess edge $N_{i t}$ and LOCOS isolation is supported by experiments on MOSFET's with non-LOCOS (etched oxide) field oxides, where a higher density of $N_{i t}$ at the field oxide edges was not observed.

\section{ACKNOWLEDGMENT}

The authors would like to thank R. Klein and R. Denton for their help with the electrical measurements, and D. McCarthy and coworkers at the NRL Nanoelectronics Processing Facility for fabrication of the NRL devices.

\section{REFERENCES}

[1] E. H. Nicollian and J. R. Brews, MOS Physics and Technology. New York, NY: Wiley, 1982.
[2] S. Wolf and R. N. Tauber Silicon Processing for the VLSI ERA, vol. 1 (Process Technology). Sunset Beach, CA: Lattice Press, 1987. pp. 296-308.

[3] J. A. Appels, E. Kooi, M. M. Paffen, J. J. H. Schatorje, and W. H. C. G. Verkulen, Philips Res. Rep., vol. 25, p. 118, 1970.

[4] Y. Tamaki, S. Isomae, S. Mizuo, and H. Higuchi, J. Electrochem. Soc., vol. 128 , p. 644, Mar. 1981

[5] J. Hui, P. Vande Voorde, and J. Moll, in 1985 Int. Electron Devices Meet. Dig., p. 392

[6] D. Chin, S. Y. Oh, S. M. Hu, R. W. Dutton, and J. L. Moll, in 1982 Int. Electron Devices Meet. Dig., p. 228.

[7] S. Wolf and R. N. Tauber, Silicon Processing for the VLSI ERA, vol. 1 (Process Technology). Sunset Beach, CA: Lettice Press, 1987, ch. 16.

[8] M. R. Chin and T. P. Ma, Appl. Phys. Lett., vol. 42, p. 883, May 1983.

[9] V. Zekeriya and T. P. Ma, IEEE Trans. Nucl. Sci., vol. NS-31, p. 1261, Dec. 1984

[10] H. E. Maes and G. Groeseneken, Electron. Lett., vol. 18, p. 372, 1982.

[11] E. Takeda, A. Shimuzu, and T. Hagiwara, IEEE Electron Device Lett., vol. EDL-4, p. 329, 1983.

[12] T. Poorter and P. Zoestbergen, in IEDM Tech. Dig., p. 100, 1984

[13] C. Plossu, C. Choquet, V. Lubowiecki, and B. Balland, Solid-State Commun., vol. 65, pp. 1231-1235, 1988.

[14] M. G. Ancona, N. S. Saks, and D. M. McCarthy, IEEE Trans. Electron Devices, vol. 35 , p. 2221 , Dec. 1988

[15] G. Groeseneken, H. E. Maes, N. Beltran, and R. F. DeKeersmaecker, IEEE Trans. Electron Devices, vol. ED-31, p. 42, Jan. 1984.

[16] N. S. Saks and M. G. Ancona, IEEE Trans. Nucl. Sci., vol. NS-34, p. 1348, Dec. 1987.

[17] P. Suciu and R. L. Johnston, IEEE Trans. Electron Devices, vol. ED27, p. 1846 , Sept. 1980.

[18] F. Hofmann and W. Krautschneider, in Proc. IEEE Conf. on Microelectronic Test Structures, vol. 2, p. 109, Mar. 1989

[19] S. Selberherr, A. Schutz, and H. W. Potzl, IEEE Trans. Electron Devices, vol. ED-27, p. 1540, 1980

[20] P. Heremans, J. Witters, G. Groeseneken, and H. E. Maes, IEEE Trans. Electron Devices, vol. 36, p. 1318, July 1989

[21] E. Arnold, J. Ladell, and C. Abowitz, Appl. Phys. Lett., vol. 13, p. 413,1989

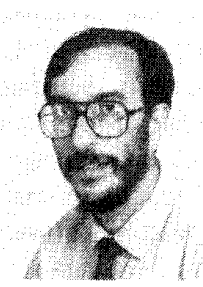

Nelson S. Saks (M'79-SM'89) received the B.A. degree in physics from Amherst College in 1968 and the M.S. degree in physics from the University of Maryland, in 1973.

He has been a research physicist in the Electronics Science and Technology Division of the Naval Research Laboratory, Washington, DC, from 1968 to the present. He has worked on amorphous semiconductors, characterization of MOS devices, development of charge-coupled devices (CCD's), and radiation effects in MOS devices. During the academic year 1984-1985, he was at the Department of Electrical Engineering at the Catholic University in Leuven, Belgium, where he worked on silicide formation and hot carrier injection in VLSI circuits. He currently serves as an advisor on MOS radiation effects to the DoD Defense Nuclear Agency. His present interests include hot carrier injection phenomena in VLSI circuits and mechanisms of radiation damage in MOS devices.

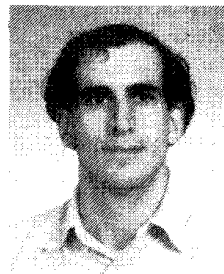

Mario G. Ancona (S'80-M'81) received the $\mathrm{Ph} . \mathrm{D}$. degree in physics mechanics at Rensselaer Polytechnic Institute, Troy, NY, in 1981.

Since that time he has been in the Electronics Science and Technology Division of the Naval Research Laboratory, Washington, DC, first as an NRC-NRL post-doctoral Research Associate and then as research physicist. His research interests have included MOS radiation and hot-carrier effects, $\mathrm{Si}-\mathrm{SiO}_{2}$ interface studies, numerical device modeling, and the theory of electron transport in 